\title{
Content and Social Network Analyses of Depression-related Tweets of African American College Students
}

\author{
Terika McCall \\ Yale School of Medicine \\ terika.mccall@yale.edu
}

\author{
Heejun Kim \\ University of North Texas \\ heejun.kim@unt.edu
}

\author{
Eun Lee \\ University of Colorado Boulder \\ eun@colorado.edu
}

\author{
Adnan Lakdawala \\ University of North Carolina at Chapel Hill \\ adnanl@live.unc.edu
}

\author{
Clinton S. Bolton III \\ University of North Carolina at Chapel Hill \\ cbolton@email.unc.edu
}

\begin{abstract}
The prevalence of depression is higher among African American college students compared to their White counterparts. They are also more likely to disclose feelings of depression on Twitter. The aim of this exploratory study was to answer the following questions: What are the most common themes of depression-related tweets among African American college students? Are there differences in the social network characteristics of college students that have posted a depression-related tweet or retweet and those who have not? Content and social network analyses were conducted. The study results showed the most common themes focused on feelings of depression, casual mentions, and supportive messages. In addition, we observed that the social networks of users posting depression-related tweets have more mutual connections with their friends than the users who did not post a depression-related tweet. These findings may help to inform the design of social media interventions for African American college students.
\end{abstract}

\section{Introduction}

The use of social media has risen in the past decade. Young adults (ages 18-29) are early adopters, and continue to use social media at high levels, with $90 \%$ using at least one social media site [1]. Twitter is one of the most popular social media sites among this group (38\% use Twitter) [1].

Due to the perception that there is some anonymity in the use of social media, and less scrutiny by parents, younger Twitter users may be more likely to communicate about sensitive issues, such as mental health, on this platform. A study by Cavazos-Rehg et al. revealed African American Twitter users were more likely to disclose feeling depressed than the average Twitter users (35\% vs. 24\%) [2]. The estimated prevalence of depression (measured by the Patient Health Questionnaire-9 depression severity score of $\geq$ 10) is higher for African American college students (17.3\%) compared to their White counterparts (15.8\%) [3]. However, how African American college students talk about depression on social media, and the social network characteristics of those who talk about depression on social media versus those who do not is understudied.

Past studies have shown that the content of tweets can signal depression, self-harm, and suicidality $[2,4]$. Findings from the Health Minds Study revealed that $67.3 \%$ of Black college students reported receiving counseling or support from a nonprofessional source (e.g., family, friends) [5]. Friends and family members were the most common sources of nonprofessional support, with $54.7 \%$ receiving support from friends, and $41.4 \%$ receiving support from family members [5]. Furthermore, social-networking sites are also a common informal way for college students to share their emotional state and seek help regarding their mental health issue [6].

Although many studies have investigated how depression is communicated on social media, to our knowledge, none have exclusively focused on this population. African American college students may communicate about depression differently than other groups. The aim of this exploratory study was to answer the following questions: What are the most common themes of depression-related tweets among African American college students? Are there any differences in the social network characteristics of African American college students that have posted a depression-related tweet or retweet on their timeline and those who have not? 


\section{Methods}

The study included both content and social network analyses. Content analysis was conducted to identify the most common themes of depression-related tweets (including retweets) among African American college students. Social network analysis was conducted to determine if there is a difference in the social network characteristics between those who posted a depressionrelated tweet or retweet on their timeline and those who did not. For the purposes of this study, the classification of African American refers to Black people who are a part of the African diaspora and reside within the United States. Hereinafter, "tweet" refers to both tweets and retweets, unless otherwise specified. Moreover, "with d$r$ tweet" refers to users who have posted a depressionrelated tweet on their timeline and "without d-r tweet" refers to users who have not posted a depression-related tweet on their timeline. The Institutional Review Board of the University of North Carolina at Chapel Hill provided the study a notification of exemption from further review.

Twitter data is publicly available, and one of the main data sources for social media analysis. Data was collected using Twitter search Application Programming Interface (API). For the implementation, we used python-twitter ${ }^{1}$, which is a Python wrapper for the Twitter API. Two datasets were collected for analyses. The first dataset was used for both content and social network analyses, and consisted of tweets from African American college students with d-r tweet. The second dataset was used for social network analysis, and consisted of random tweets from African American college students without d-r tweet.

\subsection{Dataset creation}

The first step was to identify 20 Historically Black Colleges and Universities (HBCUs) that have active Twitter followers and to find Twitter users associated with those HBCUs. HBCUs in the Southeast $(n=14)$, Northeast $(n=3)$, Southwest $(n=2)$, and Midwest $(n=1)$ United States were selected to provide a representative sample based on the geographic distribution of HBCUs. Due to historical reasons (e.g., racial segregation), most HBCUs exist in the southeast, therefore, most of the sample data was collected from Twitter users that attend a HBCU located in the southeastern United States. The name of selected HBCUs was not shared to avoid any potential negative impact, as the disclosure of the names of these schools could lead to the stigma of students that attend the schools. One of the coauthors (T.M.) collected Twitter retweet tags (e.g., @University) and hashtags (e.g., \#UniversityPride) associated with each HBCU account. Next, Twitter IDs of users who have used hashtags and retweet tags associated with each school were collected by using Twitter search API. To reduce the number of tweets collected from nonstudents (e.g., faculty, staff), users whose account description had the words such as "official", "follow us", "PhD", "Ph.D.", "Doctor", or "Postdoc"” were removed from the sample.

The second step was to create the dataset for the students with d-r tweet. Variations of the word "depression" were included in the key search terms. To capture additional search terms, T.M. searched Twitter and Google to identify common depression-related words and phrases. The 200 most recent tweets posted by each user were retrieved from their timeline, and depression-related terms ("depressed", "\#depressed", "depression", "\#depression", "dark place", "depressing", "\#depressing", "sad sad", "sadsad", "\#SadSad") were matched to those tweets. Tweets that included the depression-related terms were included in the dataset. The dataset included attributes related to tweets, such as tweet ID and tweet text, in addition to attributes related to users, such as user ID, account description, and followers' and friends' IDs. In total, data on 16 attributes were collected. The first dataset was collected between May 13, 2019 and May 15, 2019. All collected tweets were created between January 1, 2018 and May 15, 2019. A total of 1,275 tweets were collected by applying this search strategy.

The third step was to create the dataset for the students without d-r tweet. Random tweets were collected from students without d-r tweet similarly to the aforementioned process. The 200 most recent tweets posted by each user were retrieved from their timeline. However, if any of the previously listed depressionrelated terms were found in any of the user's tweets, all tweets of the corresponding user were not included in the dataset. This provided a sample of tweets from users without d-r tweet. The second dataset was collected between January 20, 2020 and January 29, 2020. We collected tweets that were created in the same period as the first dataset. A random sample of 1,649 tweets was selected to create the dataset for the students without d$r$ tweet for social network analysis only.

\subsection{Verification and filtering of tweets}

For a tweet to be included in the content and social network analyses, the user must be an African American college student attending one of the selected HBCUs at

\footnotetext{
${ }^{1}$ python-twitter.readthedocs.io/en/latest/index.html
} 
the time the tweet was created. Two members of the study team, T.M. and A.L. (health informatics graduate students), conducted independent manual verification that the users met the inclusion criteria. Race classification was decided based on the user's Twitter account profile image.

Verification of student status was based on selfreport from one or more of the following sources:

- Mention of student status in Twitter account description (e.g., University '21)

- Mention of student status in tweet (e.g., "Final exams done. I can't believe I will be graduating from college soon")

- LinkedIn profile displays that the user is currently attending one of the selected HBCUs

To determine which users and tweets should be included in the final dataset, the results of the independent verification of eligible Twitter users were compared using SPSS 26 statistical analysis software. The level of agreement between the two reviewers for each Twitter user was analyzed using Cohen's kappa [7]. Inter-coder reliability was determined by percent agreement (prevalence) and kappa coefficient. The inter-coder reliability was $80.7 \%$ agreement, kappa 0.32 . A kappa statistic of $0.21-0.40$ indicates "fair" strength of agreement [8]. Due to the relatively low kappa coefficient, only users whom both reviewers identified as meeting the inclusion criteria were included in the sample for analysis. This resulted in a sample of 682 individual users and 848 depression-related tweets for analyses. After removing duplicate tweets, the final sample included 540 depression-related tweets for content analysis. See Figure 1 for a funnel chart depicting the filtering process of depression-related tweets for the final dataset. For comparison, a sample of 682 individual users without d-r tweet was used for the social network analysis.

\subsection{Identifying themes for content analysis of depression-related tweets}

Themes identified by Cavazos-Rehg et al. [2] were used as a starting point for categorizing tweets. Additionally, grounded theory was used to produce a list of the emerging themes from review of a random sample of 100 depression-related tweets from the final dataset. T.M. and C.B. (Ph.D. and licensed clinical mental health counselor) agreed on five themes from topics that reoccurred: 1) Tweeter disclosed feeling depressed (included tweets about general feelings of depression and depression due to school-related pressures); 2) Casual mention; 3) Supportive message about depression; 4) Opinion about depression; and 5) Other. The "other" category included jokes about being depressed, mention of how people treat or talk to you about depression, information about resources or services to help with depression, suggestions on helping someone with depression. Tweets were labeled as "casual mention" if a depression-related term was arbitrarily mentioned in the tweet, however it was not the focus of the post (e.g., "Chipotle without salad dressing is depressing").

Content analysis was conducted on the 540 depression-related tweets. The tweets were imported into NVivo 12 for analysis. The tweets were then categorized by T.M. and C.B. reading through each of them and coding the emerging themes. Any disagreement over the coding of particular tweets was resolved by discussion and mutual agreement of the appropriate theme by T.M. and C.B.

\subsection{Social network analysis}

A final sample of 1,364 unique users (682 with d-r tweet, and 682 without d-r tweet) was used for the social network analysis. Friends and followers networks were created for users with d-r tweet and users without d-r tweet based on their friends and followers Twitter data. On Twitter, 'friends' means people an individual is following, and 'followers' means people who are following the individual. To express these directional relationships, directed networks were used. Each network was composed of 682 unique users identified as African American HBCU students.

Initially, each network's structural characteristics of out-degrees (friends) and in-degrees (followers) was analyzed. Then, homophily of the largest connected component of the networks was measured. The largest size of weakly connected component in a network can be measured by networkx package of 'weakly connected component' [9].

Reciprocity is another characteristic representing a mutual relationship between individuals. For instance, if an individual $i$ follows $j$ and $j$ also follows $i$, this relationship can be thought as reciprocal one. Individual-level reciprocity was measured using Eq. 1. In the equation, $A$ represents an adjacency matrix of a directed network, and $A_{j i}=1$ if $j$ follows $i$. Also, $\Lambda_{i}$ is a set of people $i$ follows, and $\delta$ is the kronecker delta which counts incoming links $j$ to $i . k_{i}^{\text {out }}$ means the number of friends $i$.

$$
R_{i}=\frac{\sum_{j \in \Lambda_{i}} \delta\left[1-A_{j, i}\right]}{k_{i}^{\text {out }}}
$$

The clustering coefficient is a metric to quantify how many of my friends are friends with each other [10]. In principle, in undirected social relationships, the 
clustering of an individual $i$ is the fraction of possible triangles through that individual that exist. For directed networks, the clustering is similarly defined as the fraction of all possible directed triangle [11]. With this, we can complement the reciprocity, meaning it can help us to see the mutual relationship in the high order (dyadic to triadic) relationship (i.e., the relationships between one's friends).

To see if there is a homophilic tendency in user connections, a ratio of users with d-r tweet among an individual's friends was measured. It represents simply how many of one's friends posted a depression-related tweet.

\section{Results}

\subsection{Content analysis of depression-related tweets}

The most common themes identified from the depression-related tweets are presented in Table 1. A total of 540 tweets were coded into themes. The tweets centered around the following themes: tweeter disclosed feeling depressed $(211 / 540,39 \%)$, casual mention (153/540, 28\%), supportive message about depression $(68 / 540,13 \%)$, opinion about depression $(66 / 540,12 \%)$, and other depression-related mentions (42/540, 8\%) which included jokes about being depressed, mention of how people treat or talk to you about depression, information about resources or services to help with depression, and suggestions on helping someone with depression. The theme 'Tweeter disclosed feeling depressed' comprised of general feelings of depression $(145 / 540,27 \%)$ and depression due to school-related pressures $(66 / 540,12 \%)$.

Approximately $67 \%$ (359/540) of the sample consisted of retweets. This finding may indicate that African American students are more likely to passively communicate about depression on social media. Supportive messages about depression primarily focused on resiliency, overcoming depression, and religion/spirituality.

\subsection{The number of friends and followers}

Using the friends and followers networks, the distribution of friends and followers was checked for each group (see Figure 2). The average number of friends of the group without d-r tweet is 916 (rounded to the nearest tenth) which is relatively smaller than the group's average number of followers $(1,293)$ (see Figure 2(a)). The trend of having more followers than friends was similar when we checked the median of the friends and followers. The median was 853 for the number of friends, and the median number of followers was 1,166 . For the user group with d-r tweet (see Figure 2(b)), the group users' median number of followers $(1,348)$ was also greater than the median of their friends (988). The inequality is the same for the average number of followers $(1,470)$ and friends $(1,166)$ for users in the with d-r tweet group. However, the median and average number of friends and followers for the group with d-r tweet is higher than the group without d-r tweet. In comparison, the median number of friends for the group with d-r tweet was greater (135 more friends) than the group without d-r tweet. Likewise, the median number of followers for the group with d-r tweet was greater (182 more followers) than the group without d-r tweet.

\subsection{Reciprocity and Clustering coefficient}

As mentioned in the previous section (Section 2.4), we measured the reciprocity $(R)$ for the ratio of the reciprocal links among a user's friends. When we check the average of the reciprocity, the average reciprocity is similar for two groups: 0.78 for the group without $\mathrm{d}-\mathrm{r}$ tweet and 0.79 for the group with d-r tweet. However, the group with d-r tweet had relatively skewed distribution of the reciprocity than that of the group without d-r tweet (see Figure 3). Figure 3 displays that the group with d-r tweet does not have the low reciprocal ratio and most of the users have high reciprocity ranging from 0.35 to 0.95 . With the consideration of an equal range of the number of friends from 0 to 3,000 , we found that the reciprocal ratio is leaning to high values in this group with d-r tweet. On the contrary, the reciprocity of the group without d-r effectively starts from 0.25 showing less skewed distribution than the other group. Causality could not be determined; however, the trend of more mutual relationships can be correlated with their depression sharing behaviors.

The relatively left-skewed reciprocal characteristics of users in the group with d-r tweet was also confirmed in the distribution of the clustering coefficient. In Figure 4 , users in the group with d-r tweet have a larger ratio (about 15\%) of users having larger than 0.004 clustering coefficient than that of the group without d-r tweet (approximately 5\%). Considering that the clustering coefficient can show the ratio of triadic-relationship, relatively high clustering coefficient of users in the group with d-r tweet supports that their network is based on the mutual relationship compared to the users in the group without d-r tweet. 


\subsection{Homophily}

When the network was restricted to include only users with and without d-r tweet, using the sub-network that was mentioned in Section 2, the results showed that users with more friends are more likely to have friends that posted a depression-related tweet (see Figures 5(a) and 6(a)). Interestingly, the users in the group without d-r tweet displayed a high tendency (0.59) to be connected with the users in the group with d-r tweet in their friendship networks (see Figure 5(b)). This means, on average, for each user in the group $59 \%$ of their friends have posted a depression-related tweet. However, the users in the group with d-r tweet are less likely to be connected to the users within their own group (group with d-r tweet) in this sub-network (see Figure 6(b)). The average percentage of friends with d$\mathrm{r}$ tweet is approximately $49 \%$ for this group. If we only calculate the ratio of users having more than $50 \%$ of friends that posted a d-r tweet (i.e., the ratio of area in the right half of the graph), $71.3 \%$ of the users in the group without d-r tweet have more than $50 \%$ of their friends posting a depression-related tweet versus $54.6 \%$ of users in the group with d-r tweet have more than half of their friends posting a depression-related tweet. The caveat is that this could be a biased effect since this subnetwork does not include the entire network of the users. This calls for further investigation into the relationship between users' depression-related tweet sharing behaviors and users' friends' sharing behaviors.

\section{Discussion}

To our knowledge, this exploratory study was one of the first to investigate how African American college students communicate about depression on social media, and differences between the social network characteristics of those who have posted a depressionrelated tweet or retweet on their timeline and those who have not. The results of the study showed that African American college students were more likely to passively communicate about depression on social media. Approximately $67 \%$ of the sample consisted of retweets. A study by Boyd et al. examined the conversational aspects of retweeting on twitter [12]. Boyd and colleagues found that reasons why people retweet include that the user is trying to "entertain or inform a specific audience, or as an act of curation", "make one's presence as a listener visible", "publicly agree with someone", or "validate other's thoughts" [12]. Retweeting invites people into a thread, allowing them to engage, or not, without having to directly address them [12]. This is important when discussing mental health, a topic that is stigmatized in the Black community.

The top three themes from the content analysis were that the tweeter disclosed feeling depressed (included tweets about general feelings of depression and depression due to school-related pressures), casual mention, and supportive message about depression. For example, one user tweeted the following regarding feeling depressed: "Anxiety and depression have been trying to get the best of me lately. I just pray for peace." Another user specifically mentioned depression due to school-related pressures and tweeted, "My thesis is like one big depressing cloud looming over all of my happy moments." Most students did not go into detail about their cause of depression unless it was school-related. This may indicate that they felt school-related depression and stressors are safer to discuss on social media than more personal causes of depression. Furthermore, school-related depression and stressors may be more relatable to their peers, providing more opportunity for engagement and social support.

Tweets in which a depression-related term was arbitrarily mentioned were coded as a casual mention. For example, one user tweeted, "Chipotle without salad dressing is depressing". Depression-related terms were used casually by many of the students in reference to anything from food to pop culture. This finding provides some insight into how African American college students communicate about depression on social media. Casual mentions may normalize use of depression-related terms, however it may not indicate that the tweeter is depressed. Therefore, use of depression-related terms may be normalized within this group although mental illness is stigmatized within the Black community [13]. However, younger African American adults are more likely to seek help (professional or informal) to cope with mental health problems compared to their older counterparts [14].

Supportive messages about depression was the next most common theme seen in tweets from African American college students. For example, one user tweeted, "God said your TURNAROUND is NOW. The enemy will regret ever messing with you. Your promotion is here. He's taking you from the bottom to the TOP. Your days are being without \& feeling depressed are OVER." The tweets primarily focused on resiliency, overcoming depression, and religion/spirituality. Regarding religion/spirituality, tweeters talked a lot about God helping them to overcome depression. This finding is supportive of past literature that shows that African Americans use religion/spirituality as a preferred coping mechanism to deal with mental health issues $[15,16]$.

The findings from this study showed that, on average, users in the d-r tweet group had more friends 
and followers than those without d-r tweet. The reciprocity was also higher among users in the d-r tweet group. However, regarding homophily, users in the group with d-r tweet were less likely to connect with users within their same group. In contrast, users in the group without d-r tweet were more likely to connect with users in the with d-r tweet group. All things considered, those who have mentioned depression may tend to share about the depression with their friends on social media to alleviate related stress. The direction of homophily and the fair number of supportive messages about depression (13\% of tweets) suggests that people without d-r tweets may tend to reach out to help people who tweet about depression. This finding demonstrates that online peer-to-peer support may be beneficial to African American college students. Online peer-to-peer support may help to reduce stigma, and increase access and engagement in online interventions for mental and physical wellbeing [17].

The main limitation of the study was that Twitter does not allow for easy filtering of users based on demographics, therefore, verification that users met the inclusion criteria was subjective and based on the available content in the user's Twitter profile (e.g., profile picture, account description, tweets). This may exclude users that may have been eligible for inclusion, however their race and student status could not be determined by the reviewers. In addition, African American college students at predominantly white institutions may communicate about depression differently than those who attend a HBCU. However, our methods employ a systematic search strategy that can provide unique social media sample data for analyzing depression-related tweets among African American college students and other communities.

The research question to compare social networks between the group with d-r tweets and the group without d-r tweets was raised while conducting the content analysis. Therefore, data for the group without $d-r$ tweets were collected later, as mentioned in the method section. Therefore, the change in the friendship network of both groups between two data collection points could not be applied. However, it should be noted that this study attempted to minimize potential confounding factors by collecting tweets produced during the same period.

\section{Conclusions and future directions}

African American college students use social media to disclose feeling depressed. The findings of this study demonstrated the need for additional research into the use of social media as a means of social support for depression among this population. Understanding how
African American college students communicate about depression may help to inform the design of culturallytailored mental health interventions to support those dealing with depression. Future research endeavors should include study of how the COVID-19 pandemic has affected the mental health of African American college students, and how they are using social media to communicate their emotions.

\section{Acknowledgements}

The first author was supported by funding from the National Library of Medicine's Institutional Training Grant for Research Training in Biomedical Informatics and Data Science at the Carolina Health Informatics Program (T15-LM012500) and Yale Center for Medical Informatics (T15-LM007056).

\section{References}

[1] Pew Research Center, "Social Media Fact Sheet", retrieved 18 June 2019 from https://www.pewinternet.org/factsheet/social-media/.

[2] Cavazos-Rehg PA, Krauss MJ, Sowles S, Connolly S, Rosas C, Bharadwaj M, et al., "A content analysis of depression-related Tweets", Comput Human Behav, 2016, vol. 54, pp. 351-357.

[3] Lipson SK, Kern A, Eisenberg D, Breland-Noble AM, "Mental health disparities among college students of color", J Adolesc Health, 2018, vol. 63, no. 3, pp. 348-356.

[4] Yazdavar AH, Al-Olimat HS, Ebrahimi M, Bajaj G, Banerjee T, Thirunarayan K, et al., "Semi-Supervised Approach to Monitoring Clinical Depressive Symptoms in Social Media", in Proceedings of the 2017 IEEE/ACM International Conference on Advances in Social Networks Analysis and Mining 2017' ' - ASONAM '17, New York, New York, USA, ACM Press, 2017, pp. 1191-1198.

[5] Eisenberg D, Hunt J, Speer N, Zivin K, "Mental health service utilization among college students in the United States", J Nerv Ment Dis, 2011, vol. 199, no. 5, pp. 301-308. [6] Eisenberg D, Hunt J, Speer N, "Help seeking for mental health on college campuses: review of evidence and next steps for research and practice", Harv Rev Psychiatry, 2012, vol. 20, no. 4, pp. 222-232.

[7] Cohen J, "A Coefficient of Agreement for Nominal Scales", Educ Psychol Meas, 1960, vol. 20, no. 1, pp. 37-46.

[8] Landis JR, Koch GG, "The measurement of observer agreement for categorical data", Biometrics, 1977, vol. 33, no. 1 , pp. 159-174.

[9] Networkx package for Python,https://networkx.github.io/ [10] Newman M, "Networks: An Introduction", Oxford University Press, Inc., 2010.

[11] Fagiolo G, "Clustering in complex directed networks", Phys Rev E, Stat Nonlin Soft Matter Phys, 2007, 76(2 Pt 2):026107.

[12] Boyd D, Golder S, Lotan G, "Tweet, tweet, retweet: conversational aspects of retweeting on twitter", in 
Proceedings of the 43rd Hawaii International Conference on System Sciences, IEEE, 2010, pp. 1-10.

[13] Thompson VLS, Bazile A, Akbar M, "African Americans' perceptions of psychotherapy and psychotherapists", Professional Psychology: Research and Practice, 2004, vol. 35, no. 1, pp. 19-26.

[14] Ward EC, Wiltshire JC, Detry MA, Brown RL, "African American men and women's attitude toward mental illness, perceptions of stigma, and preferred coping behaviors", Nursing research, 2013, vol. 62, no. 3, pp. 185-194.
[15] Ward EC, Heidrich SM, "African American women's beliefs about mental illness, stigma, and preferred coping behaviors", Res Nurs Health, 2009, vol. 32, no. 5, pp.480-492. [16] Conner KO, Copeland VC, Grote NK, Rosen D, Albert S, McMurray ML, et al, "Barriers to treatment and culturally endorsed coping strategies among depressed AfricanAmerican older adults", Aging Ment Health, 2010, vol. 14, no. 8, pp. 971-983.

[17] Naslund JA, Aschbrenner KA, Marsch LA, Bartels SJ, "The future of mental health care: peer-to-peer support and social media", Epidemiol Psychiatr Sci, 2016, vol. 25, no. 2, pp. 113-122.

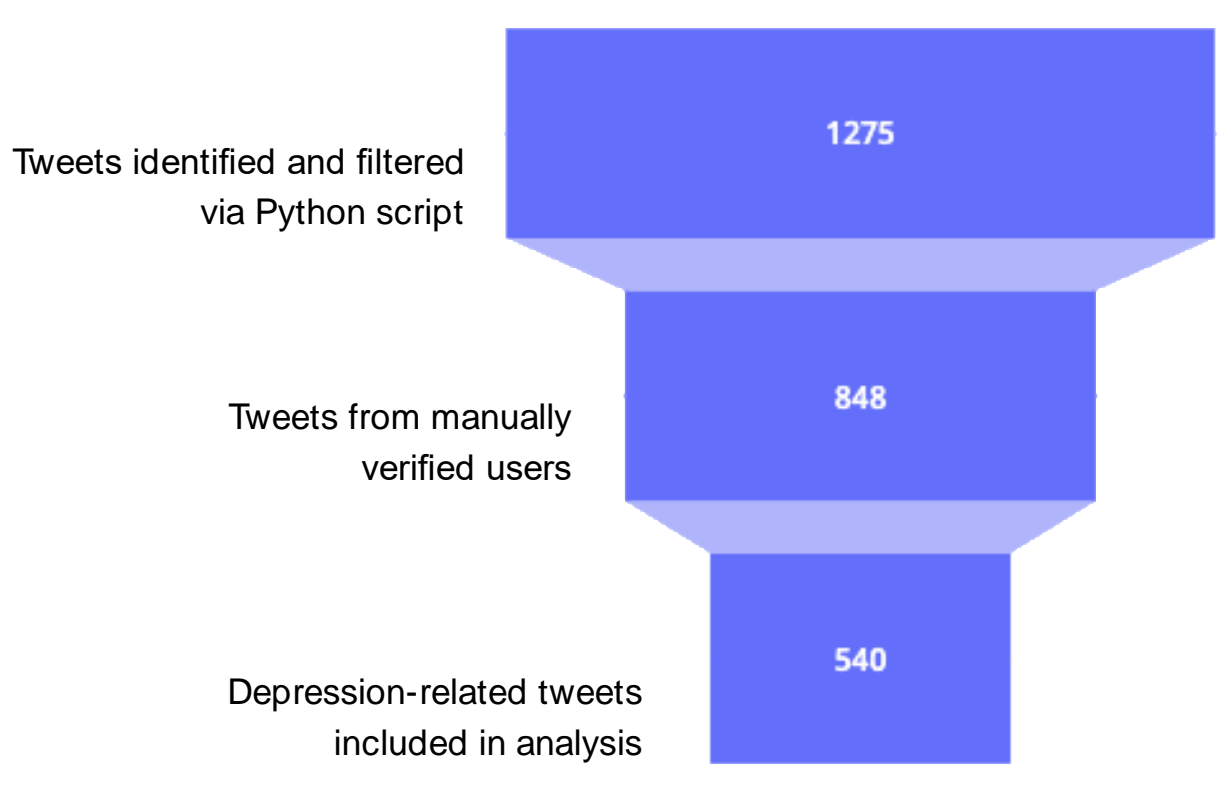

Figure 1. Funnel chart of depression-related tweets

(a)

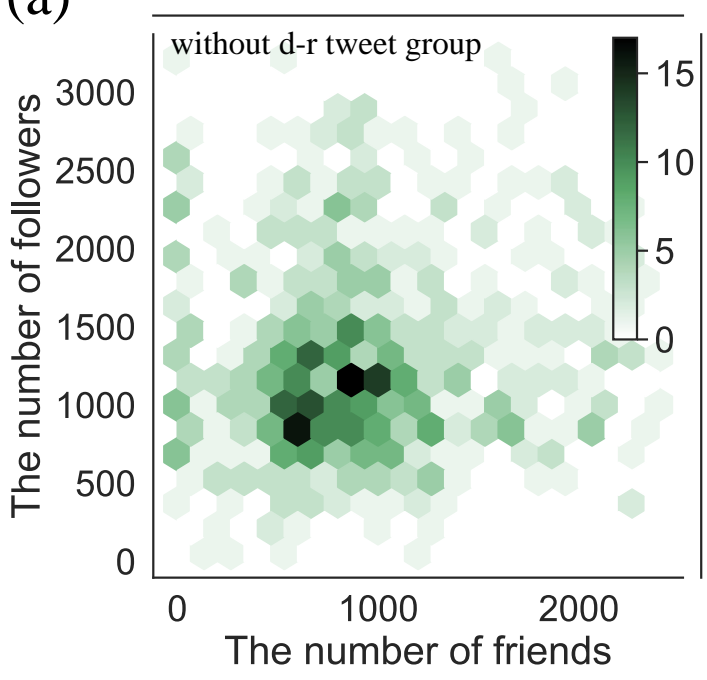

(b)

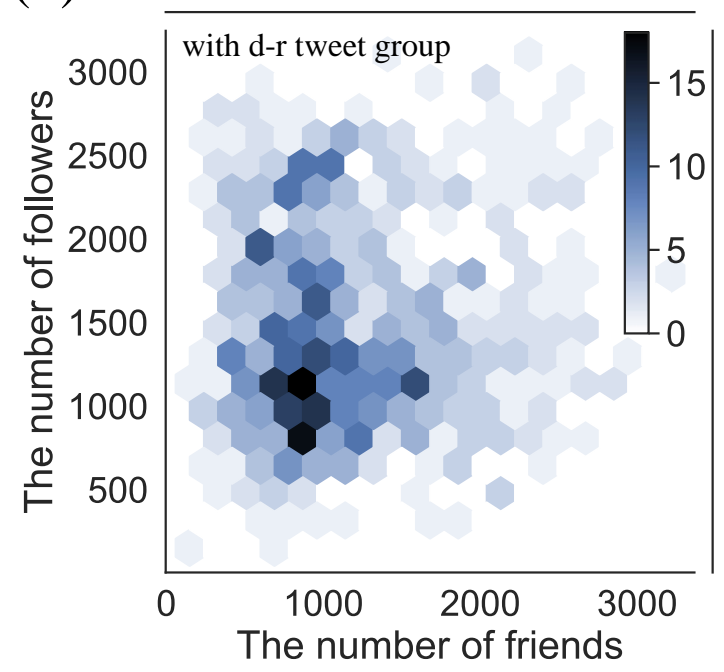

Figure 2. Distributions of the number of friends and followers of (a) the group consisting of users without a depression-related (d-r) tweet and (b) the group of users with a d-r tweet 
Table 1. Depression-related tweets of African American college students $(n=540)$

\begin{tabular}{|c|c|c|}
\hline Themes & $\mathrm{n}(\%)$ & Examples \\
\hline Tweeter disclosed feeling depressed & $211(39)$ & \\
\hline \multirow[t]{2}{*}{$\begin{array}{l}\text { General feelings of } \\
\text { depression }\end{array}$} & $\begin{array}{l}145 \\
(27)\end{array}$ & $\begin{array}{l}\text { Anxiety and depression have been trying to get the } \\
\text { best of me lately. I just pray for peace. }\end{array}$ \\
\hline & & $\begin{array}{l}R T @ \text { Twitter_handle: Yes I'm depressed I'm } \\
\text { feeling down so don't even ask } \bigcirc\end{array}$ \\
\hline \multirow[t]{2}{*}{$\begin{array}{l}\text { Depression due to school- } \\
\text { related pressures }\end{array}$} & $\begin{array}{l}66 \\
(12)\end{array}$ & $\begin{array}{l}\text { My thesis is like one big depressing cloud looming } \\
\text { over all of my happy moments. }\end{array}$ \\
\hline & & $\begin{array}{l}\text { RT @ Twitter_handle: Senior Year depression and } \\
\text { the pressure it brings is not talked about enough. I } \\
\text { use to always wonder why do people be so close to } \\
\text { the end and drop out, I TRULY get it now. I want } \\
\text { to drop out once a week. All seriousness. }\end{array}$ \\
\hline \multirow[b]{2}{*}{ Casual mention } & & Chipotle without salad dressing is depressing. \\
\hline & $153(28)$ & $\begin{array}{l}\text { RT @ Twitter_handle: my notes app be like: } \\
\text { Grocery list Future baby names Password for } \\
\text { Netflix A poem about depression FAFSA ID } \\
\text { Random sushi order }\end{array}$ \\
\hline \multirow[b]{2}{*}{$\begin{array}{l}\text { Supportive message about } \\
\text { depression }\end{array}$} & \multirow[b]{2}{*}{$68(13)$} & $\begin{array}{l}\text { Anybody battling with depression, insecurity, } \\
\text { anxiety, etc. you will get through this! You are } \\
\text { beautiful! You are amazing! And don't let nobody } \\
\text { tell you otherwise. }\end{array}$ \\
\hline & & $\begin{array}{l}\text { RT @ Twitter_handle: God said your } \\
\text { TURNAROUND is NOW. The enemy will regret } \\
\text { ever messing with you. Your promotion is here. } \\
\text { He's taking you from the bottom to the TOP. Your } \\
\text { days are being without \& feeling depressed are } \\
\text { OVER. }\end{array}$ \\
\hline \multirow[b]{2}{*}{ Opinion about depression } & \multirow[b]{2}{*}{$66(12)$} & $\begin{array}{l}\text {...depression is a debilitating disease that is } \\
\text { overpowering in someone's life they can tell if } \\
\text { that's what's wrong vs me being in a temporary } \\
\text { sadness. }\end{array}$ \\
\hline & & $\begin{array}{l}R T \text { @ Twitter_handle: A lot have depression cause } \\
\text { ya be on the internet lookin at people with these } \\
\text { fake perfect lifes, perfect body, perfect } \\
\text { relationships. Depression is hard to shake off } \\
\text { spend time with friends, closel or with yourself do } \\
\text { boards wit pictures of the things you dream make } \\
\text { them your hobby https://t.co/csOUfwzljc }\end{array}$ \\
\hline \multirow{2}{*}{ Other } & \multirow{2}{*}{$42(8)$} & $\begin{array}{l}\text { Depression looks and feels different for everyone. } \\
\text { These are common signs of depression in Black } \\
\text { Men. https://t.co/buTdwzytNK }\end{array}$ \\
\hline & & $\begin{array}{l}R T \text { @ Twitter_handle: old depression that iforgot } \\
\text { about be popping out the cut on me like "yoooo } \\
\text { roll up" }\end{array}$ \\
\hline
\end{tabular}




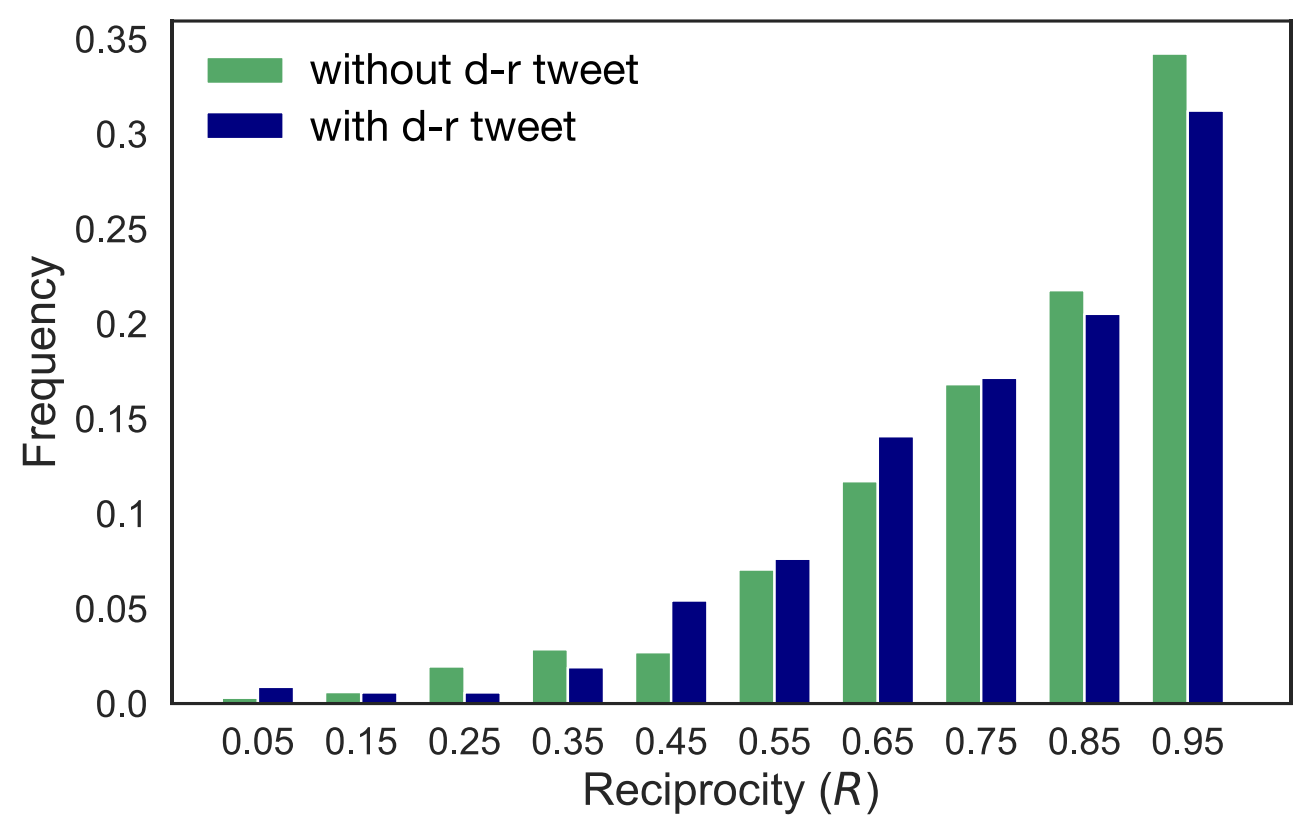

Figure 3. Distribution of the reciprocity $(R)$ of a user among the user's friends. Note: Each bin is averaged with its min and max value

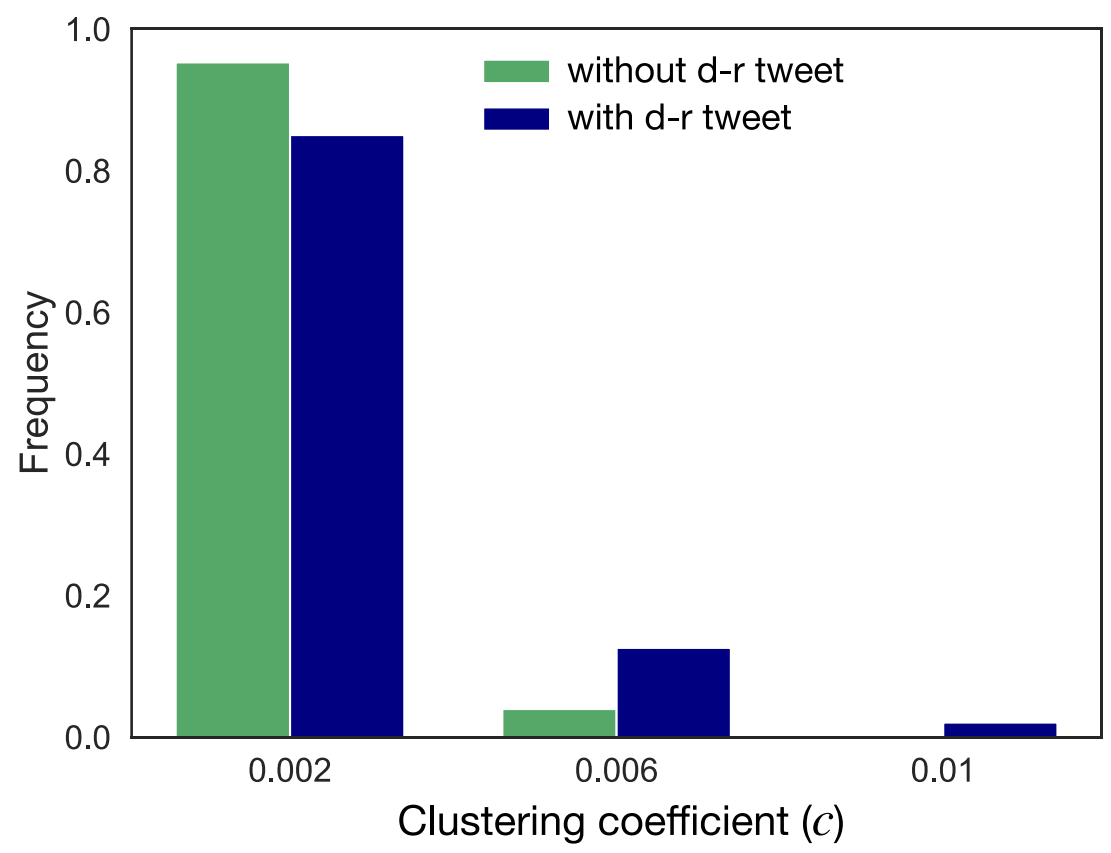

Figure 4. Distribution of the clustering coefficient of users in the group with a depression-related (d-r) tweet versus those without a d-r tweet. Note: Each bin is averaged with its min and max value 
(a)

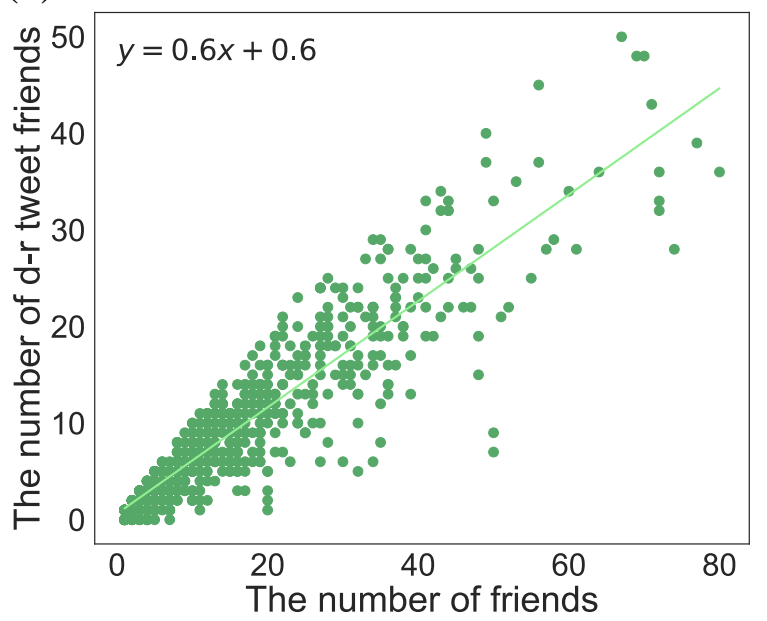

(b)

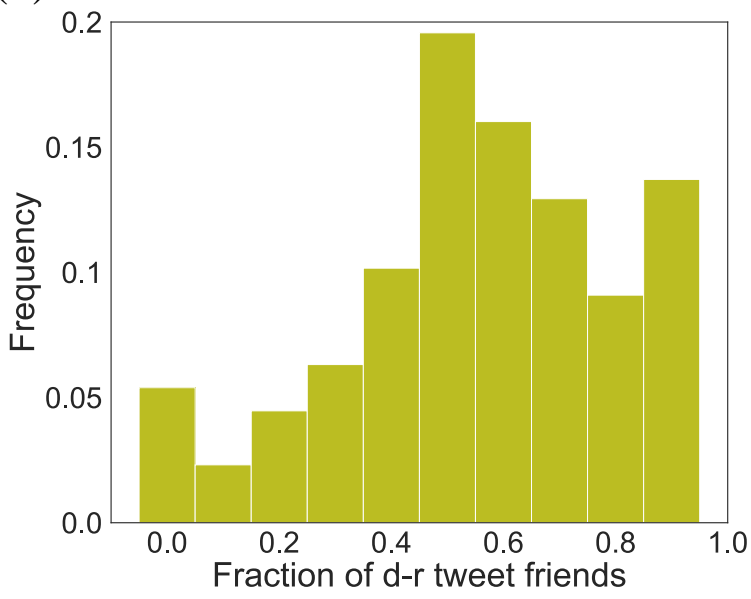

Figure 5. Homophily of the group without a depression-related tweet (d-r) (a) Scatter plot of the number of friends that posted a d-r tweet among the users' friends, (b) frequency of the users with the fraction of friends that posted a d-r tweet

(a)

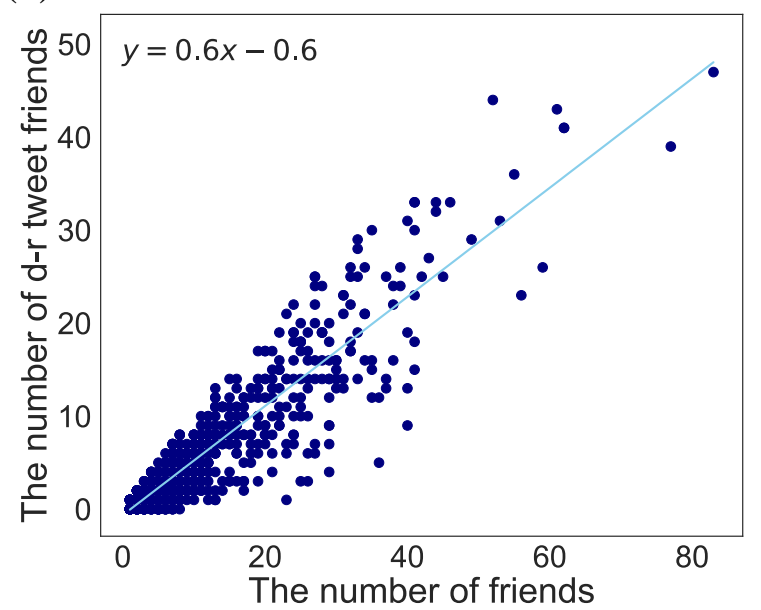

(b)

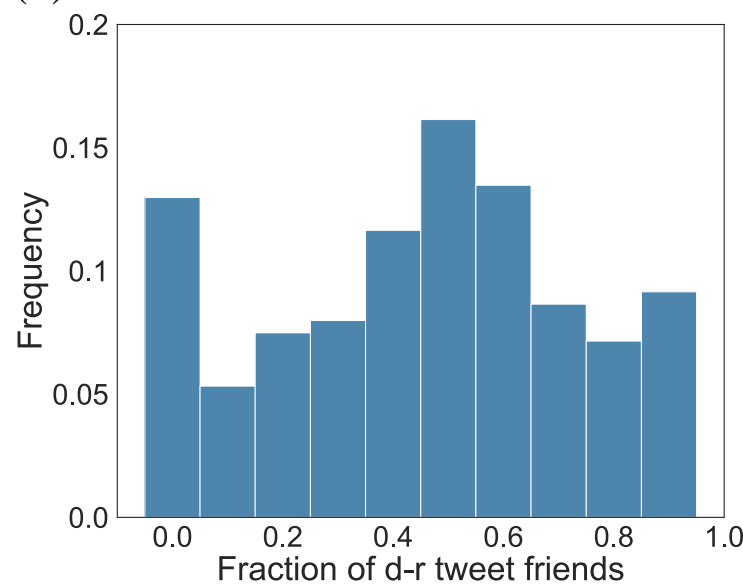

Figure 6. Homophily of the group with a depression-related tweet (d-r) (a) Scatter plot of the number of friends that posted a d-r tweet among the users' friends, (b) frequency of the users with the fraction of friends that posted a d-r tweet 